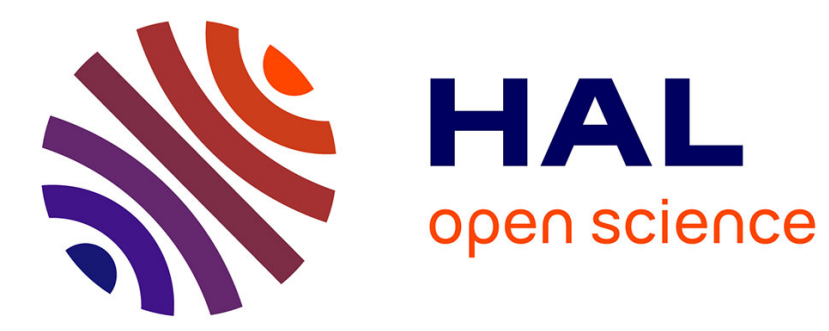

\title{
Hygro-elastic internal stresses in porous composite materials: a multi-scale analysis
}

Ziad Youssef, Frédéric Jacquemin, David Gloaguen, Ronald Guillén

\section{To cite this version:}

Ziad Youssef, Frédéric Jacquemin, David Gloaguen, Ronald Guillén. Hygro-elastic internal stresses in porous composite materials: a multi-scale analysis. Journal of Reinforced Plastics and Composites, 2008, 26 (13), pp.1417-1433. 10.1177/0731684407086610 . hal-01006936

\section{HAL Id: hal-01006936 \\ https://hal.science/hal-01006936}

Submitted on 13 Dec 2017

HAL is a multi-disciplinary open access archive for the deposit and dissemination of scientific research documents, whether they are published or not. The documents may come from teaching and research institutions in France or abroad, or from public or private research centers.
L'archive ouverte pluridisciplinaire HAL, est destinée au dépôt et à la diffusion de documents scientifiques de niveau recherche, publiés ou non, émanant des établissements d'enseignement et de recherche français ou étrangers, des laboratoires publics ou privés. 


\title{
Hygro-elastic Internal Stresses in Porous Composite Materials: A Multi-scale Analysis
}

\author{
Z. Youssef, F. Jacquemin,* D. Gloaguen And R. Guillén \\ GeM, Institut de Recherche en Génie Civil et Mécanique, UMR CNRS 6183 \\ Université de Nantes, École Centrale de Nantes, 37, Boulevard de l'Université \\ BP 406, 44602 Saint-Nazaire, France
}

\begin{abstract}
A multi-scale approach is devoted to the analysis of hygro-elastic internal stresses in porous composite materials. Voids in composite materials are known to cause significant reduction of mechanical properties. Moreover, in a humid environment they also affect the moisture absorption and the induced internal stresses. In this work, a self-consistent approach is used to estimate internal stresses induced by moisture absorption in composite materials with porous matrices. This study shows that the interaction between voids and moisture has a severe impact on the transient hygro-elastic stresses at both microscopic (fiber and matrix) and macroscopic (ply) scales.
\end{abstract}

KEY WORDS: porous composites, moisture content, hygro-elastic stresses, self-consistent model.

\section{INTRODUCTION}

$\mathbf{M}$ OISTURE ABSORPTION IN polymer matrix composites generates hygro-elastic internal stresses. These stresses are related to the intrinsic heterogeneous nature of composite materials due to the different hygroscopic swelling of the basic constituents (fiber and matrix) and adjacent plies. Multi-scale approaches have been recently developed in order to estimate the internal stresses due to hygroscopic loads at the macroscopic ply scale and at the constituent local scale. Each ply is considered as a homogenized anisotropic continuum. The displacements along a single ply are unknown and must be determined by solving the strain compatibility equations and the stress balance equations $[1,2]$. In previous studies, the corresponding stresses occurring at the micro-scale are deduced by extending the Eshelby-Kröner self-consistent model [3-5] or Mori-Tanaka approach [6,7] to hygro-elastic loads. However, these studies consider a perfect composite material without voids: the material is assumed to be perfect. In reality, the manufacturing process of composite materials induces defects such as voids in the matrix. The presence of voids, even at very low volume fraction in polymer matrix composites, causes significant reduction of mechanical properties such as strength and stiffness [8,9]. Consequently, it is interesting to analyze how the presence of voids interact with humid environments. 
In this study, a self-consistent approach is used to estimate the internal stresses induced by moisture absorption for composite materials with porous matrices at different scales.

An uncoupled approach is shown in this study, where the moisture content in the composite structure is first calculated to estimate the hygro-elastic stresses. The presence of voids affects the diffusion process in composite materials. For a Fickian behavior, the effective diffusion coefficient and the moisture saturation level are strongly modified. With the model proposed by Woo and Piggot [10], it is possible to predict the effective diffusion coefficient $D_{\text {eff }}$ linked to the diffusion coefficient of the constitutive matrix $D_{m}$ and to the void content. In our study, the diffusion process is assumed to follow Fick's law and the moisture content is calculated for void contents up to $5 \%$. Specimens with low void contents display classical Fickian behavior [9].

The mechanical macroscopic properties of the composite are obtained by considering the self-consistent model, the properties of the constituents, and the void content in the porous matrix. This approach reveals that the effective hygro-elastic properties (elastic stiffnesses and coefficients of moisture expansion) are strongly affected by the void content.

The internal stresses at the ply scale are calculated by using the classical equations of solid mechanics. The corresponding local stresses in the constituents (fibers and matrix) are deduced by using a self-consistent approach.

Thus, this study highlights the influence of voids on the transient hygro-elastic stresses. The simulations show that the increase of the void content may induce a change on the sign of stresses (from compressive to tensile state) revealing the strong interaction between porosity and humidity.

\section{CALCULATION OF THE EFFECTIVE DIFFUSION COEFFICIENT}

\section{Model of Woo and Piggott}

The aim of this section is to calculate the effective diffusion coefficient for a composite material containing voids. Woo and Piggott [10] used an electrical analogy model to calculate the effective diffusion coefficient. Indeed, transport of electrons and transport of molecules are, to a large extent, governed by the same laws. Thus, the diffusivity (reciprocal of resistivity $R$ ) for molecules is treated in the same way as conductivity for electrons.

A simple composite model, in which fibers are packed in a square array, is considered. The representative unit cell, involving a quarter of a fiber cross-section, is presented in Figure 1(a), where $a$ is the thickness of the square unit cell, $t$ is the thickness of the interphase between fiber and matrix, and $r$ is the fiber radius. $\Delta y$ is a thickness element $(0 \leq y \leq r) . R_{m}, R_{i}$, and $R_{f}$ are, the resistances of the matrix, interphase, and fiber respectively, $W_{m}, W_{i}, W_{f}$ are the thicknesses of the matrix, interphase, and fiber, respectively. For $y \leq r$, the thicknesses are:

$$
\left\{\begin{array}{l}
W_{m}=a-\sqrt{(r+t)^{2}-y^{2}} \\
W_{i}=\sqrt{(r+t)^{2}-y^{2}}-\sqrt{r^{2}-y^{2}} \\
W_{f}=\sqrt{r^{2}-y^{2}} .
\end{array}\right.
$$


(a)
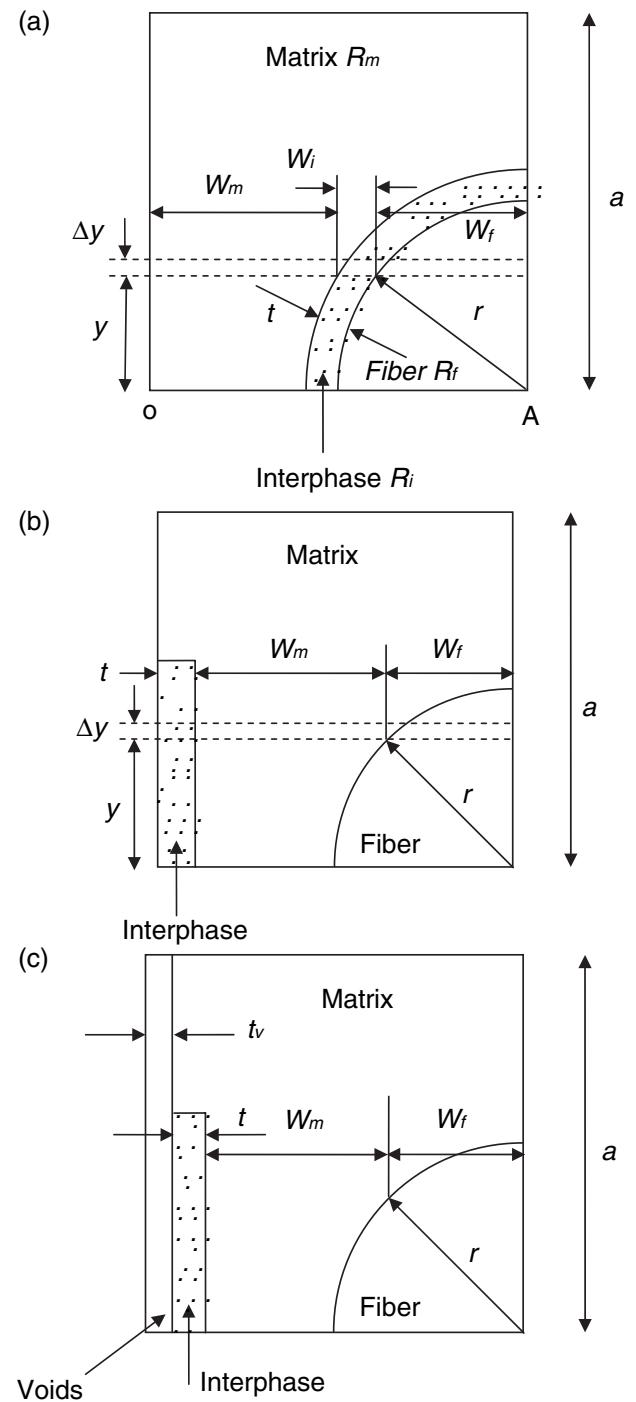

Figure 1. (a) Unit cell used for electrical analogy [10], (b) simplified unit cell, (c) unit cell with voids.

The fiber volume fraction $v^{f}$ relates the square size $a$, to the fiber diameter $2 r$ by the expression:

$$
\frac{r}{a}=2 \sqrt{\frac{v^{f}}{\pi}}
$$

For a current passing from the bottom to the top of the cell, each element, from $y=0$ to $\mathrm{y}=r$, has three resistances in parallel. Thus, the resistance $\Delta R$ for the element of thickness $\Delta y$ is:

$$
\Delta R=\Delta y\left[\frac{W_{m}}{R_{m}}+\frac{W_{i}}{R_{i}}+\frac{W_{f}}{R_{f}}\right]^{-1} .
$$


Then, the resistance in the part of the unit cell delimited by $0 \leq y \leq r$ is obtained:

$$
R=\int_{0}^{r}\left[\frac{W_{m}}{R_{m}}+\frac{W_{i}}{R_{i}}+\frac{W_{f}}{R_{f}}\right]^{-1} \mathrm{~d} y .
$$

To obtain the total resistance in the unit cell, the resistance of the region $y=r$ to $y=$ $r+t$ (where there is no fiber) and the resistance of the region $y=r+t$ to $y=a$ (where there is only the matrix) are added.

Considering that the diffusion coefficient is the reciprocal of the resistance, Woo and Piggot have finally expressed the effective diffusion coefficient $D_{\text {eff }}$ in dimensionless form:

$$
\frac{D_{\text {eff }}}{D_{m}}=\left[D_{m}\left(I D_{1}+I D_{2}\right)+1-\frac{(r+t)}{a}\right]^{-1}
$$

where:

$$
\begin{aligned}
& I D_{1}=\int_{0}^{r}\left[\left(D_{i}-D_{m}\right) \sqrt{\left(r+t^{2}\right)-y^{2}}+\left(D_{f}-D_{i}\right) \sqrt{r^{2}-y^{2}}+D_{m} a\right]^{-1} \mathrm{~d} y \\
& \left.I D_{2}=\frac{\left[\pi / 2-\sin ^{-1}(r / r+t)-\left(2 / \sqrt{1-c^{2}}\right)\left(\tan ^{-1} \sqrt{1-c / 1+c}-\tan ^{-1}\left(\sqrt{1-c / 1+c} t^{\prime}\right)\right)\right]}{\left(D_{i}-D_{m}\right)}\right\} . \\
& c=\frac{(r+t)\left(D_{i}-D_{m}\right)}{a D_{m}} \text { and } t^{\prime}=\tan \left[\frac{1}{2} \sin ^{-1}\left(\frac{r}{r+t}\right)\right] .
\end{aligned}
$$

\section{Model of Woo and Piggot in Porous Composite Media}

The diffusion coefficient of the interphase is usually unknown. Experiments indicate that it is higher than that of the matrix one [11]. The results obtained by Woo and Piggot [10] show that, for $D_{i}>10 D_{m}$, the diffusion is controlled by the ratio $t D_{i} / r D_{m}$ rather than $D_{i} / D_{m}$ and $t / r$ independently. Consequently, the authors propose to separate the interphase from the fiber, as shown in Figure 1(b).

Considering the simplified unit cell (Figure 1(b)), $W_{m}, W_{i}$, and $W_{f}$ become:

$$
\left.\begin{array}{rl}
W_{m} & =a-t-\sqrt{r^{2}-y^{2}} \\
W_{i} & =t \\
W_{f} & =\sqrt{r^{2}-y^{2}} .
\end{array}\right\} .
$$

By using Equation (5), we obtain:

$$
\frac{D_{\text {eff }}}{D_{m}}=\left[\frac{D_{m}}{D_{m}-D_{f}}\left\{\frac{2}{\sqrt{1-c^{2}}} \tan ^{-1} \sqrt{\frac{1+c}{1-c}-\frac{\pi}{2}}\right\}+\frac{D_{m} t}{D_{m}(a-t)+D_{i} t}+\frac{a-r-t}{a}\right]^{-1}
$$


with

$$
c=\frac{r\left(D_{m}-D_{f}\right)}{D_{m}(a-t)+D_{i} t} .
$$

Assuming that the diffusion coefficient of voids is also high, the same approach is used to introduce the voids in the unit cell (Figure 1(c)). $t_{v}$ represents the width of voids and $D_{v}$ their diffusivity.

Finally, the diffusion coefficient of porous composite media is:

$$
\frac{D_{\mathrm{eff}}}{D_{m}}=\left[\frac{D_{m}}{D_{m}-D_{f}}\left\{\frac{2}{\sqrt{1-c^{2}}} \tan ^{-1} \sqrt{\frac{1+c}{1-c}}-\frac{\pi}{2}\right\}+\frac{D_{m} t c}{\left(D_{m}-D_{f}\right) r}+\frac{D_{m}(a-r-t)}{D_{m}\left(a-t_{v}\right)+D_{v} t_{v}}\right]^{-1}
$$

with

$$
c=\frac{r\left(D_{m}-D_{f}\right)}{D_{m}\left(a-t-t_{v}\right)+D_{i} t+D_{v} t_{v}} .
$$

\section{Numerical Applications}

The effective diffusion coefficient is calculated by considering the unit cell depicted in Figure 1(c) without interphase $(t=0)$. The fiber volume fraction is $60 \%$ and the assumed diffusion coefficient of the voids is $D_{v}=10 D_{m}$. By assuming that the fiber does not absorb any moisture (case of carbon fiber for instance): $D_{f}=0$.

Figure 2 shows the variation of the ratio $D_{\text {eff }} / D_{m}$ as function of void content. Table 1 illustrates the values of $D_{\text {eff }} / D_{m}$ calculated for different void contents.

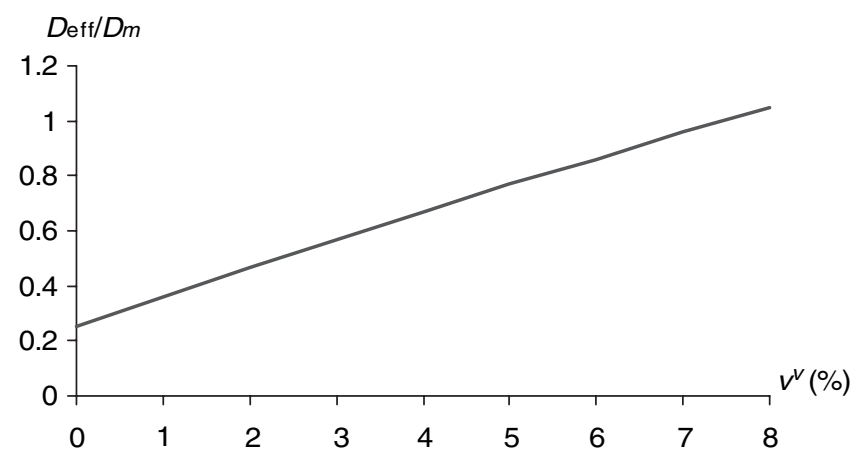

Figure 2. Evolution of $D_{\text {eff }} / D_{m}$ as function of void content.

Table 1. Values of $D_{\mathrm{eff}} / D_{m}$ as function of void content.

\begin{tabular}{cccccccccc}
\hline $\boldsymbol{v}^{\mathbf{v}}(\%)$ & $\mathbf{0}$ & $\mathbf{1}$ & $\mathbf{2}$ & $\mathbf{3}$ & $\mathbf{4}$ & $\mathbf{5}$ & $\mathbf{6}$ & $\mathbf{7}$ & $\mathbf{8}$ \\
\hline$D_{\text {eff }} / D_{m}$ & 0.25 & 0.36 & 0.47 & 0.57 & 0.67 & 0.77 & 0.86 & 0.96 & 1.05 \\
\hline
\end{tabular}


We notice obviously that the ratio $D_{\text {eff }} / D_{m}$ for a porous material is higher than a material without voids. The presence of voids in a composite material accelerates the diffusion process. Figure 2 shows the quasi-linear variation of $D_{\text {eff }} / D_{m}$ where each 0.1 increase of this ratio corresponds to $1 \%$ increase of voids. With $8 \%$ voids, the diffusion coefficient of the composite is higher than the matrix one (Figure 2). However, for such high void contents, deviations from Fickian behavior could be observed [9].

\section{PREDICTION OF THE HOMOGENIZED HYGRO-ELASTIC PROPERTIES OF A PLY}

A self-consistent (SC) model is used in order to determine the homogenized hygroelastic properties of a ply. We consider a hygro-elastic orthotropic behavior and the Eshelby-Kröner self-consistent model [5].

The multi-scale hygro-elastic orthotropic behavior can be written as:

$$
\sigma^{\alpha}=L^{\alpha}:\left(\varepsilon^{\alpha}-\beta^{\alpha} \Delta C^{\alpha}\right)
$$

where $L^{\alpha}$ is the elasticity tensor of the phase $\alpha, \varepsilon$ the strains due to elastic and hygroscopic solicitations, $\beta$ the coefficients of moisture expansion (CME), and $\Delta C$ the moisture content increment.

The macroscopic stresses and strains (the macroscopic scale is denoted by the superscript ${ }^{I}$ ) are the volume averages of the microscopic stresses and strains [12]:

$$
\left.\begin{array}{c}
\left\langle\sigma^{\alpha}\right\rangle_{\alpha=f, m, v}=\sigma^{I} \\
\left\langle\varepsilon^{\alpha}\right\rangle_{\alpha=f, m, v}=\varepsilon
\end{array}\right\}
$$

where $f, m$, and $v$ are respectively the subscripts $\alpha$ for the fiber, the matrix, and the voids.

By using Eshelby's formalism, we obtain the following relation between macroscopic and microscopic fields [5]:

$$
\varepsilon^{\alpha}=\left(L^{\alpha}+L^{I}: R^{I}\right)^{-1}:\left[\left(L^{I}+L^{I}: R^{I}\right): \varepsilon^{I}+L^{\alpha}: \beta^{\alpha} \Delta C^{\alpha}-L^{I}: \beta^{I} \Delta C^{I}\right]
$$

where $R^{I}$ represents the reaction tensor that expresses the elastic interactions due to the morphology assumed for the elementary constituents of the composite material.

The combination of Hill's average relation (10) with the preceding equations gives:

$$
\varepsilon^{I}=\left\langle\left(L^{\alpha}+L^{I}: R^{I}\right)^{-1}:\left[\left(L^{I}+L^{I}: R^{I}\right): \varepsilon^{I}+L^{\alpha}: \beta^{\alpha} \Delta C^{\alpha}-L^{I}: \beta^{I} \Delta C^{I}\right]\right\rangle_{\alpha=f, m, v} .
$$

The equation develops as:

$$
\begin{aligned}
\varepsilon^{I}= & \left\langle\left(L^{\alpha}+L^{I}: R^{I}\right)^{-1}:\left(L^{I}+L^{I}: R^{I}\right)\right\rangle_{\alpha=f, m, v}: \varepsilon^{I} \\
& +\left\langle\left(L^{\alpha}+L^{I}: R^{I}\right)^{-1}:\left[L^{\alpha}: \beta^{\alpha} \Delta C^{\alpha}-L^{I}: \beta^{I} \Delta C^{I}\right]\right\rangle_{\alpha=f, m, v} .
\end{aligned}
$$


Since this relation must be satisfied for any hygro-mechanical state $\left\{\varepsilon^{\mathrm{I}}, \Delta C^{\mathrm{I}}, \Delta C^{f}, \Delta C^{m}\right.$, $\left.\Delta C^{v}\right\}$, the first term of the right member of Equation (13) must be equal to $I$, while the second term must be nil:

$$
\begin{aligned}
& \left\langle\left(L^{\alpha}+L^{I}: R^{I}\right)^{-1}:\left(L^{I}+L^{I}: R^{I}\right)\right\rangle_{\alpha=f, m, v}=I \\
& \left\langle\left(L^{\alpha}+L^{I}: R^{I}\right)^{-1}:\left[L^{\alpha}: \beta^{\alpha} \Delta C^{\alpha}-L^{I}: \beta^{I} \Delta C^{I}\right]\right\rangle_{\alpha=f, m, v}=0 .
\end{aligned}
$$

Equation (14) yields the macroscopic elastic stiffness:

$$
L^{I}=\left\langle\left(L^{\alpha}+L^{I}: R^{I}\right)^{-1}:\left(L^{I}+L^{I}: R^{I}\right): L^{\alpha}\right\rangle_{\alpha=f, m, v}
$$

Equation (16) shows that the presence of voids modifies the macroscopic elastic properties. Knowing that the elastic tensor of voids is nil, this modification is just related to the void volume fraction.

The development of Equation (15) gives:

$$
\left\langle\left(L^{\alpha}+L^{I}: R^{I}\right)^{-1}: L^{\alpha}: \beta^{\alpha} \Delta C^{\alpha}\right\rangle_{\alpha=f, m, v}=\left\langle\left(L^{\alpha}+L^{I}: R^{I}\right)^{-1}: L^{I}: \beta^{I} \Delta C^{I}\right\rangle_{\alpha=f, m, v} .
$$

Since, $L^{I}, \beta^{I}$, and $\Delta C^{I}$ are constants, we may extract them from the summation:

$$
\left\langle\left(L^{\alpha}+L^{I}: R^{I}\right)^{-1}\right\rangle_{\alpha=f, m, v}^{-1}:\left\langle\left(L^{\alpha}+L^{I}: R^{I}\right)^{-1}: L^{\alpha}: \beta^{\alpha} \Delta C^{\alpha}\right\rangle_{\alpha=f, m, v}=L^{I}: \beta^{I} \Delta C^{I}
$$

Finally, an explicit form for the macroscopic CME of a porous composite material is obtained:

$$
\beta^{I}=\frac{1}{\Delta C^{I}} L^{I}-1:\left\langle\left(L^{\alpha}+L^{I}: R^{I}\right)^{-1}\right\rangle_{\alpha=f, m, v}^{-1}:\left\langle\left(L^{\alpha}+L^{I}: R^{I}\right)^{-1}: L^{\alpha}: \beta^{\alpha} \Delta C^{\alpha}\right\rangle_{\alpha=f, m, v}
$$

Since the increment of moisture content in fiber and the elastic tensor of voids are nil, we obtain the simplification:

$$
\beta^{I}=v^{m} \frac{\Delta C^{m}}{\Delta C^{I}} L^{I}-1:\left\langle\left(L^{\alpha}+L^{I}: R^{I}\right)^{-1}\right\rangle_{\alpha=f, m, v}^{-1}:\left(L^{m}+L^{I}: R^{I}\right)^{-1}: L^{m}: \beta^{m}
$$

where $v^{m}$ stands for the volume fraction of the matrix in the considered ply $\left(v^{m}+v^{f}+v^{v}=1\right)$.

The moisture content of the composite as a function of the moisture contents of the resin and voids is given by the expression [13]:

$$
\Delta C^{I}=\frac{v^{m} \rho^{m} \Delta C^{m}+v^{e} \rho^{e}}{\rho^{I}}
$$


Table 2. Mechanical properties of T300/5208 constituents.

\begin{tabular}{lccccc}
\hline Material & $E_{\mathbf{1}}(\mathrm{GPa})$ & $E_{\mathbf{2}}, \boldsymbol{E}_{\mathbf{3}}(\mathrm{GPa})$ & $\boldsymbol{v}_{\mathbf{1 2}}, \boldsymbol{v}_{\mathbf{1 3}}$ & $\mathbf{G}_{\mathbf{2 3}}$ (GPa) & $\mathbf{G}_{\mathbf{1 2}}$ (GPa) \\
\hline T300 & 230 & 15 & 0.2 & 7 & 15 \\
N5208 & 2.9 & 2.9 & 0.35 & 1.07 & 1.07 \\
\hline
\end{tabular}

Table 3. Hygroscopic properties of T300/5208 constituents.

\begin{tabular}{lcc}
\hline Material & $\boldsymbol{\beta}_{\mathbf{1}}$ & $\boldsymbol{\beta}_{\mathbf{2}}$ \\
\hline T300 & 0 & 0 \\
N5208 & 0.6 & 0.6 \\
\hline
\end{tabular}

Table 4. Hygroscopic expansion coefficients as function of void content.

\begin{tabular}{lccc}
\hline $\boldsymbol{v}^{\boldsymbol{v}}(\%)$ & $\boldsymbol{\Delta} \boldsymbol{C}^{\boldsymbol{m}} / \boldsymbol{\Delta} \boldsymbol{C}^{\prime}$ & $\boldsymbol{\beta}_{\mathbf{1}}$ & $\boldsymbol{\beta}_{\mathbf{2}}$ \\
\hline 0 & 3.31 & 0.02 & 0.88 \\
1 & 2.49 & 0.01 & 0.65 \\
2 & 1.99 & 0.012 & 0.51 \\
3 & 1.66 & 0.009 & 0.42 \\
4 & 1.42 & 0.008 & 0.35 \\
5 & 1.24 & 0.007 & 0.30 \\
\hline
\end{tabular}

where $\rho^{I}, \rho^{m}$, and $\rho^{e}$ are the composite, matrix, and water densities, respectively; $v^{e}$ represents the volume fraction of voids filled with water.

Finally, we obtain the macroscopic CME of the composite:

$$
\beta^{I}=\frac{v^{m} \rho^{I} \Delta C^{m}}{v^{m} \rho^{m} \Delta C^{m}+v^{e} \rho^{e}} L^{I^{-1}}:\left\langle\left(L^{\alpha}+L^{I}: R^{I}\right)^{-1}\right\rangle_{\alpha=f, m, v}^{-1}:\left(L^{m}+L^{I}: R^{I}\right)^{-1}: L^{m}: \beta^{m}
$$

The effect of void content on the hygroscopic properties of the composite T300/5208 is studied. The mechanical and hygroscopic properties of the carbon fiber T300 and epoxy matrix are summarized in Tables 2 and $3[14,15]$.

Table 4 illustrates the values of the $\Delta C^{m} / \Delta C^{I}$ ratio in relation to the saturation of the composite, as well as the values of the hygroscopic expansion coefficients as function of void content. We note that the water density $\rho^{e}$ is $1000 \mathrm{~kg} / \mathrm{m}^{3}$, the matrix and composite ones are, respectively, 1200 and $1590 \mathrm{~kg} / \mathrm{m}^{3}$.

Figure 3 shows the variation of the transverse CME as function of void content. Contrary to the effective diffusion coefficient, the hygroscopic expansion coefficients decrease with the growth of the void volume fraction. Then, the presence of voids in the composite involves an accelerated diffusion process along with a less important hygroscopic expansion of the material. 


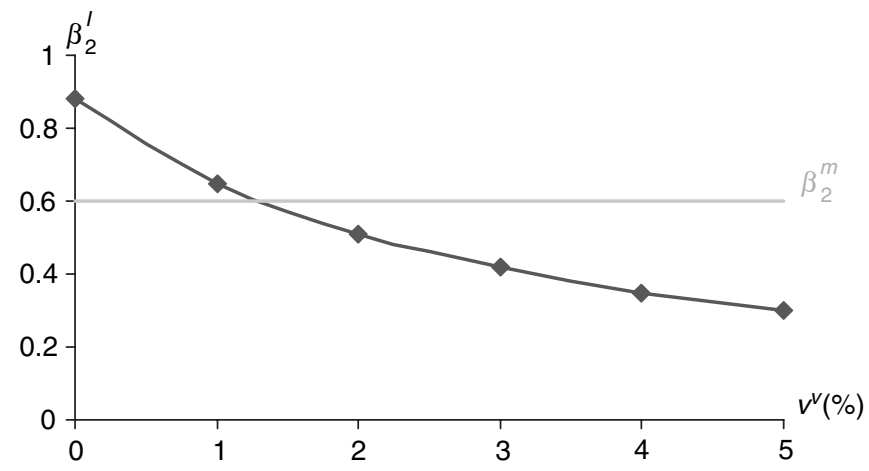

Figure 3. Variation of the transverse CME as function of void content.

\section{MOISTURE CONTENT}

\section{Macroscopic Moisture Contents}

Consider an initially dry, $4 \mathrm{~mm}$ thin composite pipe, where inner and outer radii are $a$ and $b$, respectively, and let the laminate be exposed to an ambient fluid with boundary moisture content $C_{0}$. The composite cylinder is made up of T300/5208 carbon/epoxy plies alternatively oriented at $\pm 55^{\circ}$ versus the longitudinal axis. The macroscopic moisture content, $C^{I}(r, t)$, is a solution of the following system with Fick's Equation (23), where $D_{\text {eff }}$ is the transverse effective diffusion coefficient deduced from Woo and Piggot's model.

The moisture content is calculated for void contents up to $5 \%$. The values of $D_{\text {eff }}$ are extracted from Table 1 by considering a diffusion coefficient for the N5208 epoxy resin equal to $D_{m}=7.31 .10^{-8} \mathrm{~mm}^{2} / \mathrm{s}[15]$ :

$$
\left.\begin{array}{c}
\frac{\partial C^{I}}{\partial t}=D_{\mathrm{eff}}\left[\frac{\partial^{2} C^{I}}{\partial r^{2}}+\frac{1}{r} \frac{\partial C^{I}}{\partial r}\right], \quad a<r<b \\
C^{I}(a)=C^{I}(b)=C_{0} \\
C^{I}(r, 0)=0
\end{array}\right\} .
$$

Figure 4 depicts the macroscopic moisture content profiles inside the composite pipe without voids. After 200 months, a uniform saturation level of $1.67 \%$ (corresponding to an external relative humidity of $100 \%$ ) is reached.

When the void content is $5 \%$, the macroscopic moisture content at saturation is equal to $4.73 \%$ (Figure 5) and the time to reach the saturation is 70 months (the effective diffusion coefficient for $5 \%$ voids is three times higher).

To make a comparison between the different void contents, the average of the moisture contents (25) is considered as a function of square root of time. With this representation, 


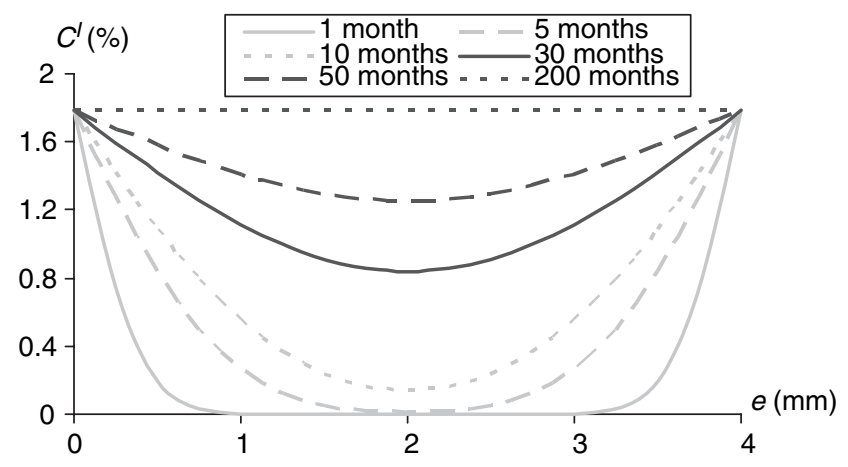

Figure 4. $T 300 / 5208$ moisture content profiles for $v^{v}=0 \%$.

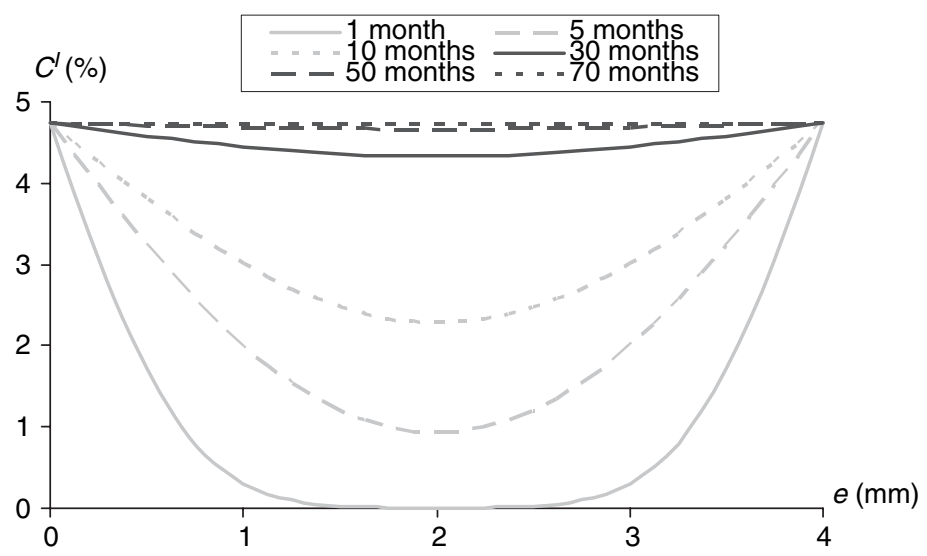

Figure 5. T300/5208 moisture content profiles for $v^{v}=5 \%$.

it is conceivable to compare the moisture contents at saturation and the diffusion coefficients.

$$
C^{t}=\frac{1}{e} \int_{0}^{e} C^{I}(r, t) \mathrm{d} r .
$$

Figure 6 shows, up to the saturation level, a linear evolution (Fickian behavior) of the moisture contents as a function of square root of time. The presence of voids in the composite involves an accelerated diffusion process (the slope is a growing function of the void content) and higher moisture saturation level.

\section{Moisture Contents in the Resin and Voids}

To determine, in the next part of the article, the transient local stresses, we need to know the moisture contents in the resin and voids. The methodology proposed in this study is based on the stability of the CME during the diffusion process whatever the void content. The coefficient of moisture expansion $\beta^{I}$ is conventionally defined as the free expansion 


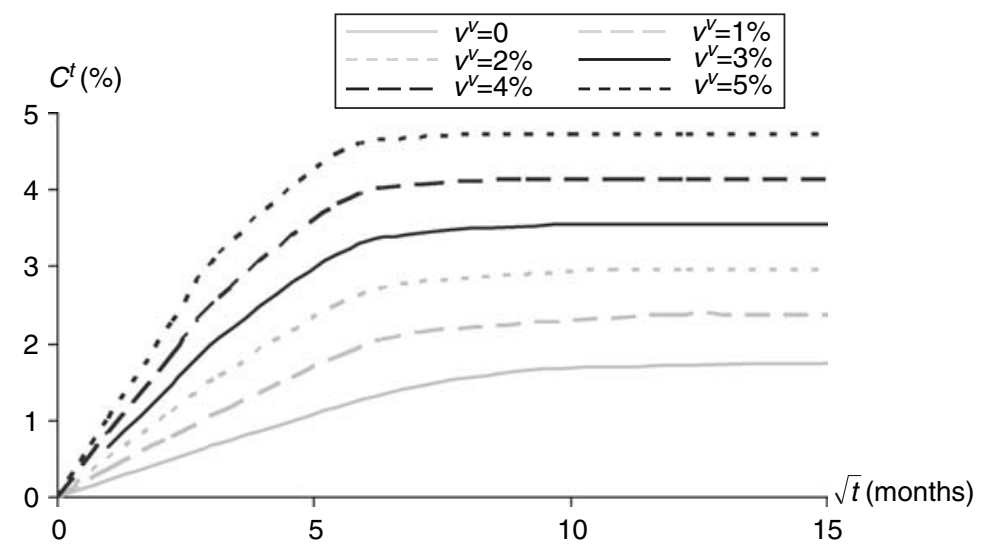

Figure 6. Average moisture contents as function of void content.

hygroscopic strain of a unidirectional laminate $\varepsilon_{\text {sat }}^{H}$ induced by absorbed average moisture content $C_{\text {sat. }}^{I}$ at saturation:

$$
\beta^{I}=\frac{\varepsilon_{\text {sat. }}^{H}}{C_{\text {sat. }}^{I} .}
$$

In this study, the coefficient of moisture expansion $\beta^{I}$ is defined as the free expansion hygroscopic strain of a unidirectional laminate $\varepsilon^{H}$ induced by absorbed average moisture content $C^{I}$ at time $t$ (during the transient pattern):

$$
\beta^{I}=\frac{\varepsilon^{H}}{C^{I}}
$$

Jedidi et al. [16] have experimentally studied the hygrothermal strain as a function of moisture content up to the saturation state for several unidirectional carbon/epoxy specimens. The authors emphasize the linear evolution of the hygroscopic strain $\varepsilon^{H}$ versus the moisture content $C^{I}$ for each specimen. Therefore, $\beta^{I}$ can be considered as constant during the diffusion process whatever the time.

Knowing the value of $\beta^{I}$ and the macroscopic moisture content $\Delta C^{I}$, Equation (20) leads to the moisture content in the resin $\Delta C^{m}$ for each ply at every time. Figure 7 shows the moisture content profiles in the matrix for a $5 \%$ void content. The time to reach saturation for the matrix with $5 \%$ voids is three times faster compared with the matrix without void.

The next step consists of estimating, during the diffusion process, the transient moisture content in the voids for each ply of the composite structure. From the knowledge of the macroscopic and matrix moisture contents, $\Delta C^{I}$ and $\Delta C^{m}$, respectively, Equation (21) gives $v^{e}$, the volume fraction of voids filled with water.

Figure 8 shows $v^{e}$ as a function of time for a composite structure in which each ply presents a porous matrix with $5 \%$ voids. For the first time, the voids in the external plies are quasi-saturated while the voids in the central region of the structure are quasi-dried. The saturation in water of all the voids takes, as the matrix, 70 months. 


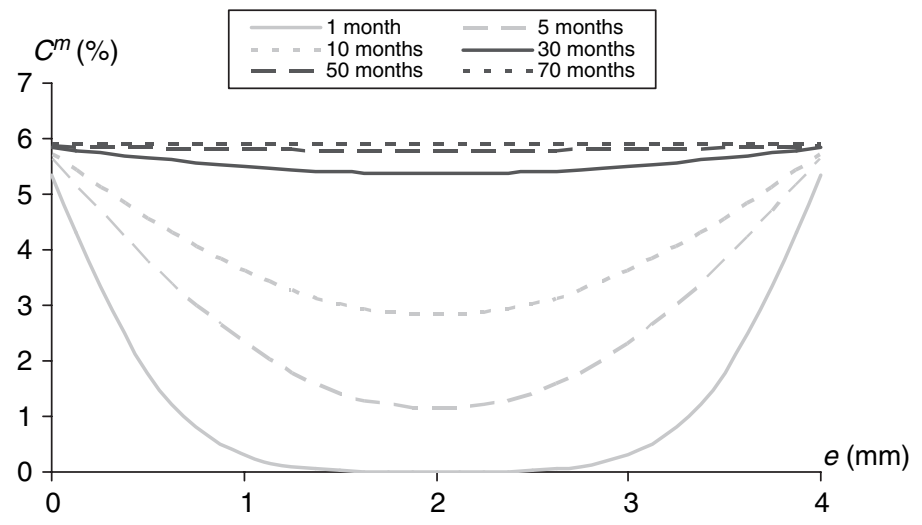

Figure 7. Moisture content in the resin for $v^{v}=5 \%$.

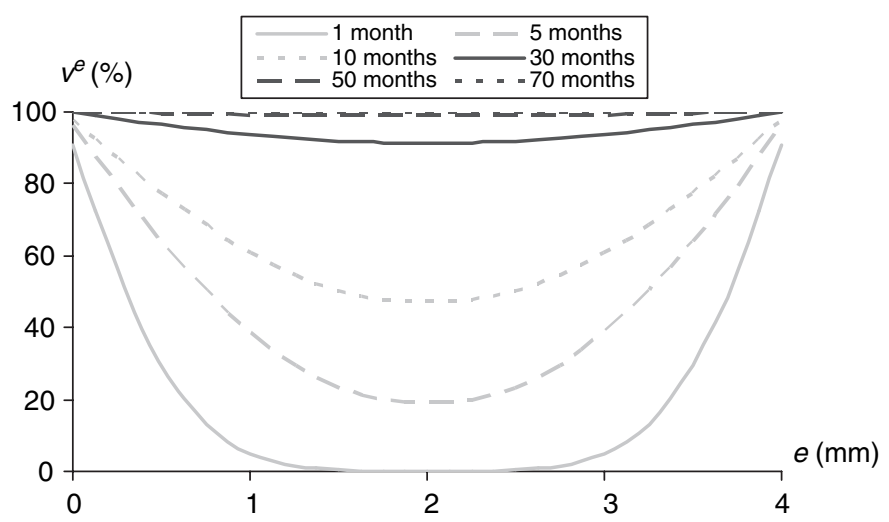

Figure 8. Moisture content in the voids for $v^{v}=5 \%$.

\section{HYGRO-ELASTIC STRESSES}

\section{Permanent Hygro-elastic Stresses}

The macroscopic hygro-elastic stresses are calculated by considering the homogenized hygro-elastic properties and the classical equations of solid mechanics: constitutive laws of hygroelastic orthotropic materials, strain-displacement relationship, compatibility and equilibrium equations, and boundary conditions (for a more detailed description of the model, see Ref. [1]).

Figures 9 and 10 show the transverse and shear stresses, respectively, when the permanent saturation state is reached in the composite structure, as a function of void content.

For the moisture permanent state, the transverse stresses are quasi-uniform over the thickness of the pipe. Compressive stresses, whatever the void content, are predicted. The transverse stresses decrease with the void content: the maximum transverse stress is $-95 \mathrm{MPa}$ for a $0 \%$ void content. The discontinuity of the shear stress is due to the 


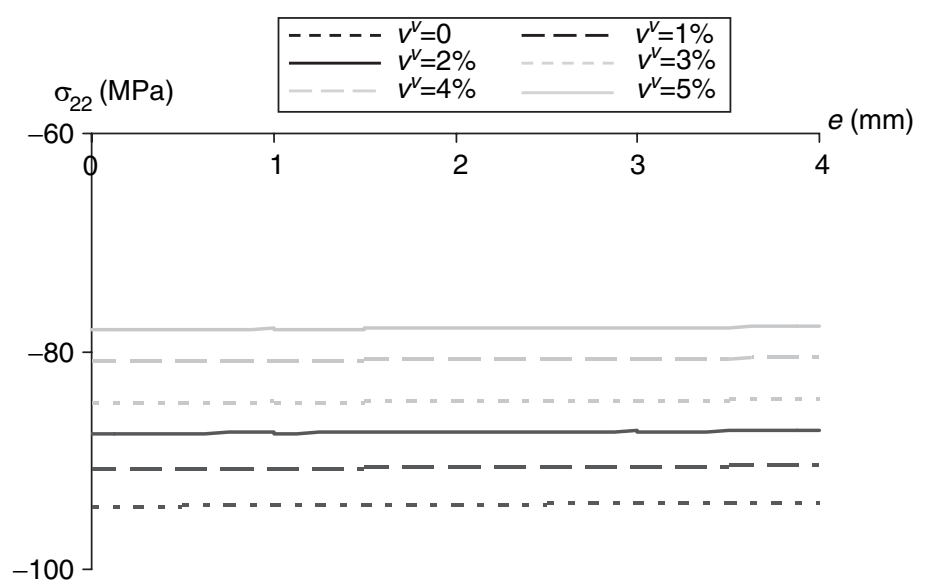

Figure 9. Permanent transverse stresses.

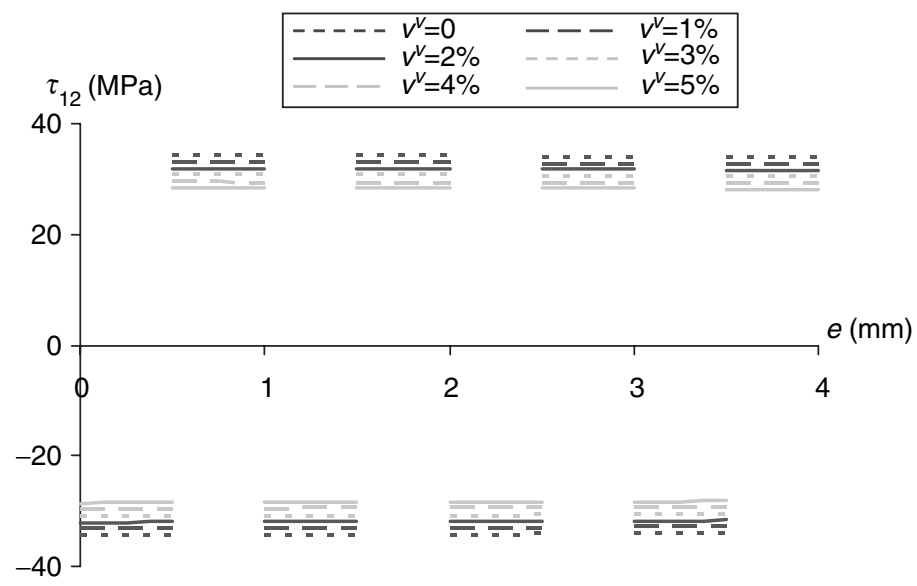

Figure 10. Permanent shear stresses.

stacking sequence: the composite pipe is made up of T300/5208 plies alternatively oriented at $\pm 55^{\circ}$ versus the longitudinal axis.

Finally, higher transverse and shear stresses hold in the structure without voids even if the moisture content is less important compared with a porous structure. The key factor is the moisture expansion coefficient which is more important for a structure without voids (Figure 3).

The corresponding local stresses are deduced by using the self-consistent approach.

By assuming that the fibers do not absorb any moisture, the local stresses-strains relation in reinforcements (9) becomes:

$$
\sigma^{f}=L^{f}: \varepsilon^{f}
$$


Using Eshelby's formalism, we obtain the following scale transition expression for the strains in fibers:

$$
\varepsilon^{f}=\left(L^{f}+L^{I}: R^{I}\right)^{-1}:\left(\sigma^{I}+L^{I}: R^{I}: \varepsilon^{I}\right)
$$

The use of Equation (29) implies the knowledge of the macroscopic stresses and strains, and the homogenized properties. If these conditions are satisfied, the local mechanical states in the epoxy matrix are provided by Hill's strains and stresses average laws (10):

$$
\left.\begin{array}{c}
\varepsilon^{m}=\frac{1}{v^{m}} \varepsilon^{I}-\frac{v^{f}}{v^{m}} \varepsilon^{f} \\
\sigma^{m}=\frac{1}{v^{m}} \sigma^{I}-\frac{v^{f}}{v^{m}} \sigma^{f}
\end{array}\right\} .
$$

Figures 11 and 12 show the multi-scale transverse and shear stresses, respectively, as functions of void content, when the permanent saturation state is reached. At permanent

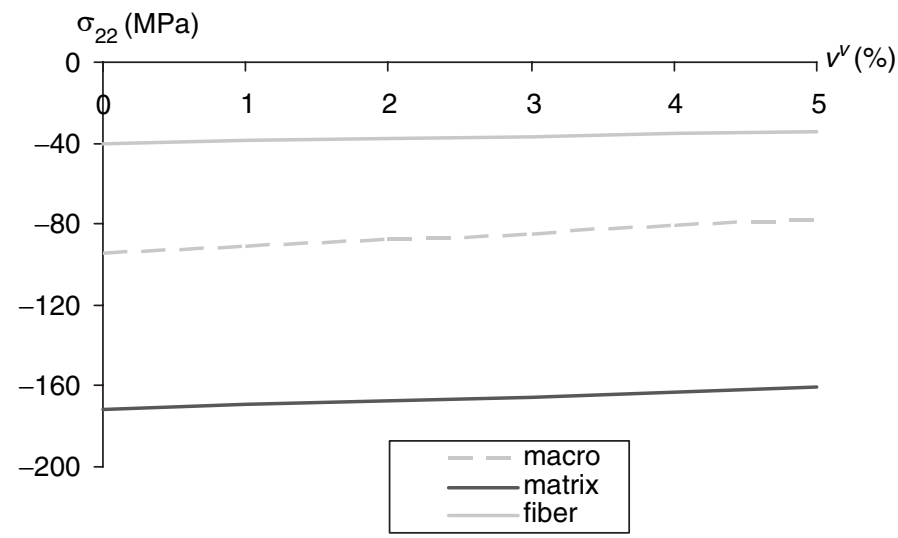

Figure 11. Multi-scale permanent transverse stresses.

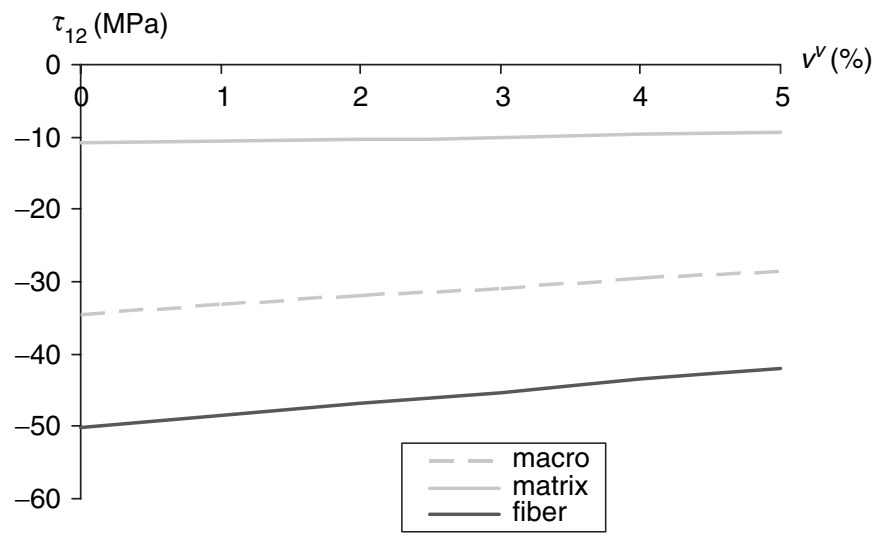

Figure 12. Multi-scale permanent shear stresses. 
state, the maximum transverse stress holds in the matrix. The fibers are submitted to the higher shear stresses. The local stresses decrease with the void content.

\section{Transient Hygro-elastic Stresses}

Figures 13 and 14 depict, respectively, the macroscopic transverse and shear stresses, after 5 months, as functions of void content. The change from a tensile to compressive state, for the central region of the pipe, emphasizes the influence of the moisture content, and thus the void content. For $0 \%$ void, the weak moisture content in the center of the pipe induces tensile transverse stresses. Nevertheless, for $5 \%$ voids, at the same time, in the same region, the moisture content is more important and the induced stresses are negative. In the external plies, whatever the void content, compressive stresses hold caused by the strong moisture content in these zones. Contrary to the transverse stresses, the shear stresses are uniform on each ply of the structure.

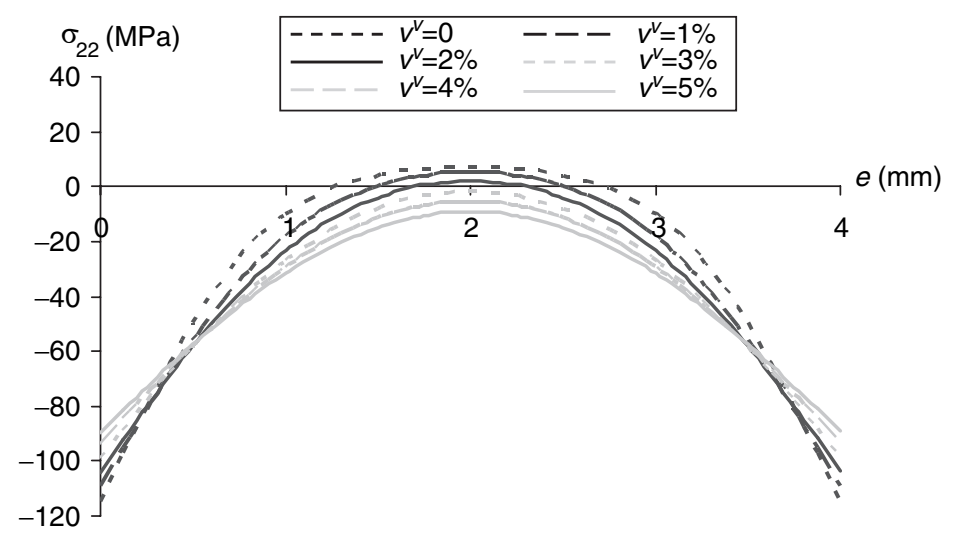

Figure 13. Transverse stress at $t=5$ months as function of void content.

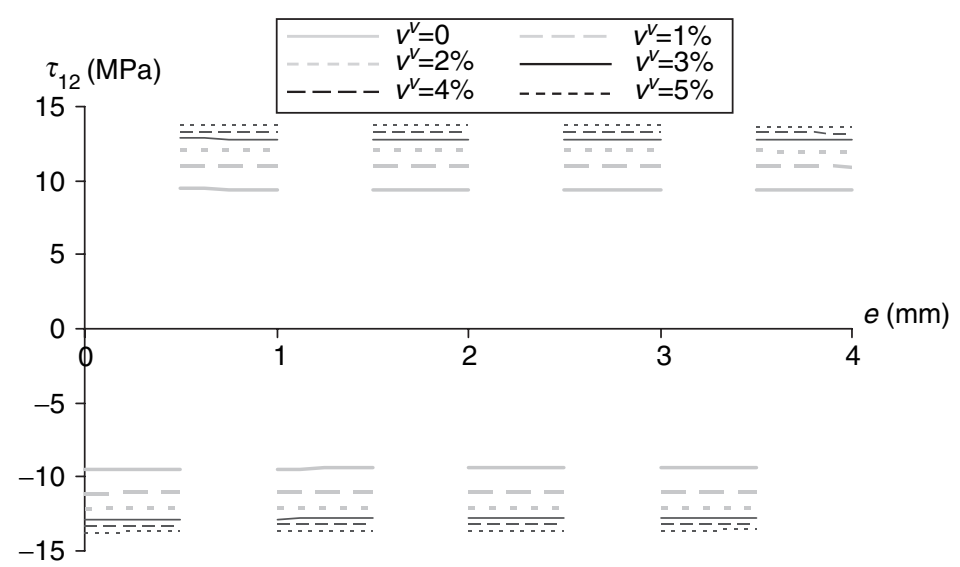

Figure 14. Shear stress at $t=5$ months as function of void content. 


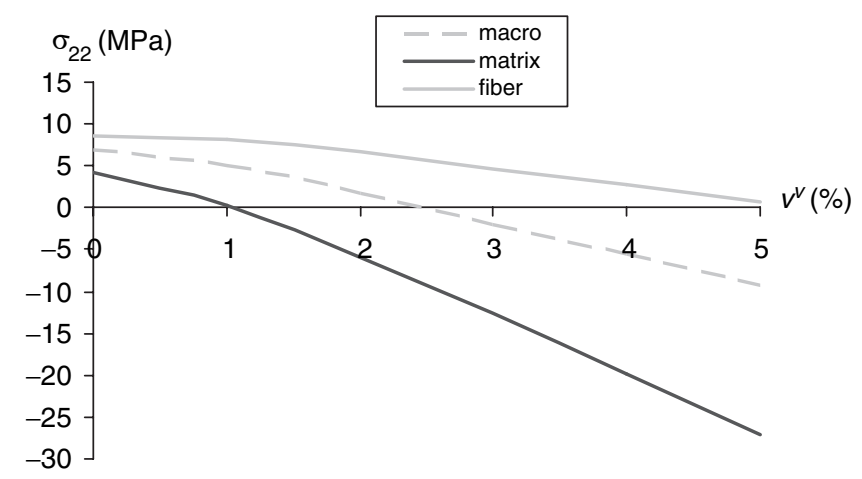

Figure 15. Multi-scale transverse stresses at $t=5$ months.

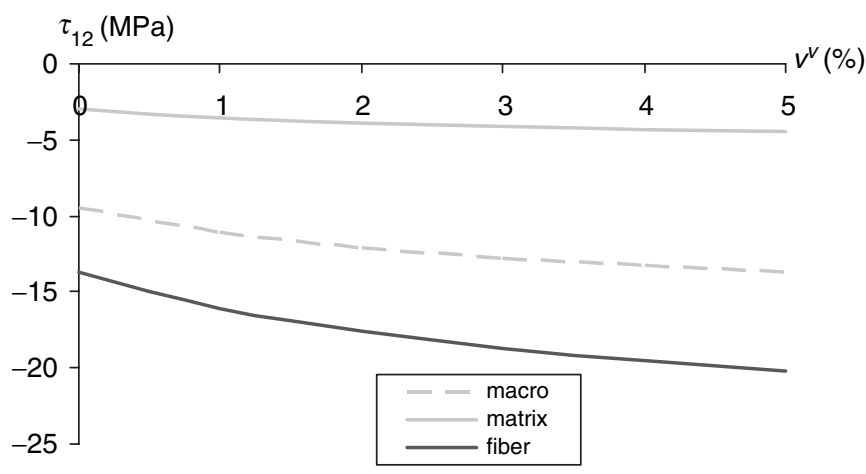

Figure 16. Multi-scale shear stresses at $t=5$ months.

Figures 15 and 16 present, respectively, the multi-scale transverse and shear stresses, during the transient state, at $t=5$ months, as functions of void content.

The amount of void content involves a change of sign for the transverse stresses in the matrix. A decrease of the tensile transverse stresses in the fibers is also observed. The compressive shear stresses in the fibers are sensitive to the void content since an increase is observed with the void content.

\section{CONCLUSIONS}

A multi-scale analysis devoted to the determination of hygro-elastic internal stresses in porous composite materials is proposed. An uncoupled approach is used to estimate the internal stresses. The moisture content in the porous composite structure is first calculated by assuming a Fickian behavior with an effective diffusion coefficient deduced from Woo and Piggot's model. A self-consistent approach is used to predict the homogenized hygro-elastic properties by modeling fibers, matrix, and voids as three distinct phases. The macroscopic stresses are calculated by using continuum mechanics formalism and the local stresses derived from the self-consistent model. The proposed approach allows us to determine the multi-scale internal stresses taking into account the interaction between 
voids contained in the matrix and humid environments. The influence of void content on the diffusion process and transient hygro-elastic stresses is emphasized, showing the strong interaction between porosity and humidity: for instance, an increase of the void content may induce a change from compressive to tensile stresses.

\section{REFERENCES}

1. Jacquemin, F. and Vautrin, A. (2002). A Closed-form Solution for the Internal Stresses in Thick Composite Cylinders Induced by Cyclical Environmental Conditions, Composite Structures, 58: 1-9.

2. Gigliotti, M., Jacquemin, F. and Vautrin, A. (2007). Assessment of Approximate Models to Evaluate Transient and Cyclical Hygrothermoelastic Stress in Composite Plates, International Journal of Solids and Structures, 44: 733-759.

3. Eshelby, J. D. (1957). The Determination of the Elastic Field of an Ellipsoidal Inclusion, and Related Problems, Proc. Roy. Soc. London, A241: 376-396.

4. Kröner, E. (1958). Berechnung der elastischen Konstanten des Vielkristalls aus des Konstanten des Einkristalls, Zeitschrift für Physik, 151: 504-518.

5. Jacquemin, F., Fréour, S. and Guillén, R. (2004). A Hygro-elastic Self-consistent Model for Fiber-reinforced Composites, Journal of Reinforced Plastics and Composites, 5: 485-502.

6. Mori, T. and Tanaka, K. (1973). Average Stress in Matrix and Average Elastic Energy of Materials with Misfitting Inclusions, Acta Metallurgica, 21(5): 571-574.

7. Fréour, S., Jacquemin, F. and Guillén, R. (2006). Extension of Mori-Tanaka Approach to Hygroelastic Loading of Fiber-reinforced Composites - Comparison with Eshelby-Kroner Self-consistent Model, Journal of Reinforced Plastics and Composites, 25: 1039-1052.

8. Huang, H. and Talreja, R. (2005). Effects of Voids Geometry on Elastic Properties of Unidirectional Fiber Reinforced Composites, Composite Science and Technology, 65: 1964-1981.

9. Harper, B. D., Staab, G. H. and Chen, R. S. (1987). A Note on the Effects of Voids Upon the Hygral and Mechanical Properties of AS4/3502 Graphite/Epoxy, Journal of Composite Materials, 21: 280-289.

10. Woo, M. and Piggot, M. R. (1988). Water Absorption of Resins and Composites: IV Water Transport in Fiber Reinforced Plastics, Journal of Composites Technology and Research, 10: 20-24.

11. Chua, P. S. (1985). Ph.D. thesis, University of Toronto, Canada.

12. Hill, R. (1967). The Essential Structure of Constitutive Laws for Metals Composites and Polycrystals, Journal of the Mechanics and Physics of Solids, 15: 79-95.

13. Tsai, S. W. and Hahn, H. T. (1980). Introduction to Composite Materials, Lancaster: Technomic Publishing Co., Inc., Pennsylvania.

14. Agbossou, A. and Pastor, J. (1997). Thermal Stresses and Thermal Expansion Coefficients of n-Layered Fiber-reinforced Composites, Composites Science and Technology, 57: 249-260.

15. Loos, A. C. and Springer, G. S. (1981). Moisture Absorption of Graphite - Epoxy Composition Immersed in Liquids and in Humid air in: Environmental Effects on Composite Materials, Technomic Publishing, pp. 34-55.

16. Jedidi, J., Jacquemin, F. and Vautrin, A. (2006). Characterisation of Hydric Expansion for Carbon/Epoxy Laminates, European Conference on Composite Materials 12th August 29 - September 1, Biarritz, France. 